\title{
Leiomyoma in the Retzius space
}

\author{
Dobrosława Sikora-Szczęśniak
}

Specialist Hospital, Radom, Poland

\begin{abstract}
Parauterine location of leiomyomas is observed to be an extremely rare clinical condition. However, when benign or malignant primary tumours are detected in that area, they are often found in the Retzius space. There have been only six cases of leiomyomas in the Retzius space reported in the literature so far. The paper presents one such leiomyoma case located in the Retzius space. This extremely rare location of the tumour justifies its presentation. The medical records of the patient treated in the outpatient's obstetrics and gynaecology (ob-gyn) clinic, her case history, and hospital records, supplemented by information obtained from the patient's daughter, were analysed. The patient, a 50-year-old woman with type 2 diabetes, was referred to hospital for diagnosed secondary anaemia due to heavy menstruation for four months, which was associated with intramural leiomyoma in the anterior wall of the uterine body. Clinical examination found a large leiomyoma located in the anterior wall of the uterus. Laparotomy was performed: apart from and a leiomyoma in the uterine body it found a tumour, $140 \times 100 \mathrm{~mm}$ in size, in the Retzius space. Total resection of the tumour was performed. Since the leiomyoma was located in the uterine wall, hysterectomy with bilateral excision of adnexa was also performed. The patient was discharged home in generally good condition on the sixth day following the operation. Concomitant occurrence of leiomyoma atypically located in the Retzius space and leiomyoma in the uterine body made perioperative diagnosis very difficult.
\end{abstract}

Key words: leiomyoma in the Retzius space.

\section{Introduction}

Benign tumours located in the extraperitoneal space occur with $15-20 \%$ frequency, and among them $1.2 \%$ are leiomyomas $[1,2]$. Leiomyomas located in the extraperitoneal space are most often found in the pelvis minor [3].

The Retzius space is a part of the extraperitoneal space, between the pubic symphysis and the urinary bladder, often referred to as retropubic or antero-vessical, paravesical and pararectal, retropubic space, cave of Retzius, space of Retzius, or Retzius cavity. The Retzius space is located between transversalis fascia of the abdominal wall and the parietal peritoneum [4].

The Retzius space a place where benign tumours and primary malignancies are rarely diagnosed in women $[5,6]$. There have been only six cases of leiomyomas in the Retzius space reported in the literature so far [2, 7-10].

The paper presents a case of atypical location of leiomyoma in the Retzius space.

The paper presents a case history of the patient with leiomyoma in the Retzius space. No complications were reported over a 10-year period after surgery. In addition, the patient's medical records from the out- patient ob-gyn clinic and information provided by the patient's daughter were analysed.

\section{Case description}

The patient, a 50-year-old woman with type 2 diabetes, was admitted to hospital in severe distress on 27.09.2000. The patient was referred to hospital with diagnosed secondaryanaemia(HCT-18.3\%, RBC-2.0M/ $\mu$, $\mathrm{HGB}-5.1 \mathrm{~g} / \mathrm{dl}$ ) due to massive blood loss during menstruation for four months. Heavy bleeding was associated with a myoma in the uterine body. The patient reported a history of urinary complaints, difficult and irregular frequency of urination, and haematuria prior to hospitalisation. The following were recorded on admission: blood pressure $-155 / 70 \mathrm{mmHg}$, pulse $-106 /$ min, temperature $-36.8^{\circ} \mathrm{C}$, body weight $-73 \mathrm{~kg}$, BMI $-29.2 \mathrm{~kg} / \mathrm{m}^{2}$.

Clinical examination and trans-vaginal USG found a large leiomyoma, approximately $100 \mathrm{~mm}$ in diameter, located in the anterior wall of the uterus. The patient was operated on. Apart from the leiomyoma, laparotomy revealed a tumour, $140 \times 100 \mathrm{~mm}$ in size, located in the Retzius space. The tumour was totally resected. 
Additionally, hysterectomy with bilateral excision of adnexa was performed because the leiomyoma was intramurally located.

Histopathological findings:

- tumour in the Retzius space - leiomyoma oedematosum hyalinisans.

- leiomyoma in the uterine body - leiomyoma intramurale partim cellulare partim hyalinisans.

The patient died 10 years later due to cardio-vascular complications caused by diabetes.

\section{Discussion}

Parauterine location of leiomyomas is defined as an extremely rare clinical condition. Pepe et al. noted only five cases of leiomyomas in the Rezius space reported in the literature so far [10]. Another case was described by Niva et al. [2].

Leiomyomas detected in the extraperitoneal space occur with $73 \%$ frequency in the pelvis minor [7], and $<1 \%$ leiomyomas are found in the broad ligament of the uterus, which is thought to be an extremely rare location [11, 12].

The researchers present various theories on the aetiology of leiomyomas developing in the extraperitoneal space. Kho et al. suggested iatrogenic aetiology. They observed that $83 \%$ of leiomyomas located extraperitoneally and intraperitoneally, with no evident connection to the uterus, were diagnosed in the patients with a history of abdominal operations, and $67 \%$ of patients underwent myomectomies by laparoscopy with morcellation [13]. Other authors think that extraperitoneal leiomyomas originate from the remains of the Müllerian or Wolffian ducts or from the smooth muscles cells of the vascular walls [7, 14]. In $40 \%$ of cases retroperitoneal leiomyomas co-exist with uterine leiomyomas or can be related to past hysterectomy $[3,15]$.

Precise location of lesions in the pelvis minor, or in the intra- or extraperitoneal space is essential for future treatment, especially if operation is considered [4]. To precisely locate a new growth in the extraperitoneal space, imaging diagnostics is of basic importance. CT scans usually visualise it as a homogenous mass. MRI scans show it as a mas of low T2 signal and medium T1, and signal of various intensity on contrast scans [14]. Diagnostic transdermal biopsy and histopathological examination may help preoperatively to precisely locate lesions [2].

In the presented case preoperative diagnosis of the lesion was difficult because the tumour was atypically located in the Retzius space and co-existed with a leiomyoma of the uterus. The initial causative relationship of diagnosed anaemia only to the leiomyoma in the uterine body was wrong because uterine bleedings were also observed in cases of extraperitoneal tumours [16]. In addition, it turned out that preoperative diag- nostics limited to USG prevented proper diagnosis because in the case of tumours located extraperitoneally MRI is the most useful technique [2, 4, 14]. Moreover, correct diagnosis was more difficult due to the fact that tumours are rarely located extraperitoneally, and relating them to urinary complaints were not considered well enough. Complaints such as dysuria, haematuria, anuria, and irregular and frequent urination were noted in the reported cases of leiomyomas in the Retzius space $[8,10]$.

Preoperative precise differentiation between extraperitoneal tumours and malignancies such as leiomyosarcomas pose great clinical difficulty [2]. In the presented case of leiomyoma in the Rezius space histopathological examination found oedema and hyaline degeneration.

In the case of leiomyomas located in the broad ligament, degenerations result from insufficient blood supply. Those often take the form of myxomatoses, and rarely calcifications [16].

Definitive diagnosis of leiomyoma is based on the histopathological results. If ambiguous, additional immunohistochemical tests are required.

Surgical management of extraperitoneal leiomyomas involves total resection with or without hysterectomy by laparotomy. In the case of leiomyoma in the Retzius space described above, hysterectomy with bilateral excision of adnexa was performed.

\section{Conclusions}

In the case of urinary complaints and differential diagnosis of problems in the pelvis minor, benign tumours in the extraperitoneal cavity should be considered, including the Retzius space. Imaging diagnostics, especially MRI if the mass is located extraperitoneally, are essential to make correct preoperative diagnosis and to establish the best surgical treatment plan.

\section{Disclosure}

The authors report no conflict of interest.

\section{References}

1. Jemal A, Thomas A, Murray T, et al. Cancer statistics. CA J Clin 2002; 52: 23-47.

2. Niwa $\mathrm{N}$, Yanaihara $\mathrm{H}$, Horinaga $M$, et al. Leiomyoma in Retzius space: an unusual location. Can Urol Assoc J 2013; 7: E612-613.

3. Poliquin V, Victory R, Vilos GA. Epidemiology, presentation, and management of retroperitoneal leiomyomata: systematic literature review and case report. J Minim Invasive Gynecol 2008; 15: 152-160.

4. Shanbhogue AKP, Fasih N, Macdonald DB, et al. Uncommon primary pelvic retroperitoneal masses in adults: a pattern-based imaging approach. Radiographics 2012; 32: 795-817.

5. Tan $\mathrm{CH}$, Vikram R, Boonsirikamchai P, et al. Pathways of extrapelvic spread of pelvic disease: imaging findings. Radiographics 2011; 31: 117-133. 
6. Payne S, Adair R, Alvarez J, et al. Aggressive angiomyxoma of the space of Retzius: a case report. J Low Genit Tract Dis 2003; 7: 304-306.

7. Stutterecker D, Umek W, Tunn R, et al. Leiomyoma in the space of Retzius: a report of 2 cases. Am J Obstet Gynecol 2001; 185: 248-249.

8. Reisenauer C, Walz-Mattmueller R, Solomayer EF, et al. Leiomyoma in the Retzius space: a rare cause for voiding difficulties. Int Urogynecol J Pelvic Floor Dysfunct 2007; 18: 1229-1231.

9. Molina Granados JF, Alegre Catellanos FA, Escribano Fernandez J. Leiomioma gigante del espacio de Retius. Cirugia Espanola 2010; 88: 49-50.

10. Pepe F, Pepe P, Rapisarda F, et al. Giant leiomyoma of the Retzius space: a case report. Case Rep Obstet Gynecol 2013; 2013: 371417.

11. Bakari F, Sulayman HU, Avidime S, et al. Huge broad ligament leiomyoma: a case report. Case Reports in Clinic Med 2015; 4: 55-59.

12. Chmaj-Wierzchowska K, Buks J, Wierzchowski M, et al. Leiomyoma cellulare in the broad ligament of the uterus - case report and review of literature. Ginekol Pol 2012; 83: 301-304.

13. Kho KA, Nezath C. Parasitic myomas. Obstet Gynecol 2009; 114: 611-5.

14. Fasih N, Shanbhogue AKP, Macdonald DB, et al. Leiomyomas beyond the uterus: unusual locations, rare manifestations. Radiographics 2008; 28: 1931-1948.

15. Watanabe K, Tanaka K. Mesometrial smooth muscle as an origin of female retroperitoneal (pelvic) leiomyomas. Virchows Arch 2007; 451: 899-904.

16. Godbole RR, Lakshmi KS, Vasant K. Rare case of giant broad ligament fibroid with myxoid degeneration. J Sci Soc 2012; 39: 144-146. 